\title{
Tuberculosis pulmonar en los trabajadores de salud del Hospital Nacional Dos de Mayo de Lima
}

\author{
Pulmonary tuberculosis in health care workers in \\ Dos de Mayo National Hospital, Lima
}

\author{
Carlos Contreras,' Alberto Cortez ${ }^{2}$ y Humberto Lira ${ }^{3}$
}

Contreras C, Cortez A, Lira H. Tuberculosis pulmonar en los trabajadores de salud del Hospital Nacional Dos de Mayo de Lima. Rev Soc Peru Med Interna. 2019;32(I):15-20.

\begin{abstract}
RESUMEN
Овлетіvo. Determinar el perfil epidemiológico de trabajadores de salud (TS) del Hospital Nacional Dos de Mayo (HNDM) de Lima, Perú, afectados de tuberculosis pulmonar (TP). Material y métodos. Estudio observacional, descriptivo y retrospectivo. La población consistió de TS con diagnóstico de TP. Se recolectaron variables epidemiológicas: edad, sexo, comorbilidad, grupo ocupacional, servicio o departamento al cual pertenece, situación laboral, tiempo de servicio, entre otras. Para las variables cuantitativas se determinó la media, desviación estándar, rango, valor inferior y superior, para las variables cualitativas se utilizó el análisis de frecuencia. Se determinaron tasas de incidencia por 100000 , frecuencias absolutas y relativas, pruebas estadísticas de tendencia central y de dispersión. Se consideró el valor de $p<$ 0,05 como significativo. Resultados. La tasa de incidencia habitual de TP fue de 348 por 100000 . El grupo más afectado fue el personal técnico auxiliar (23,96\%). El promedio de edad fue 35,52 años ( $D E \pm 10,22), 70,83 \%$ fueron menores de 40 años. El tiempo promedio de trabajo hospitalario fue de 9,23 años (DE $\pm 7,32$ ). 91,70\% tuvieron tiempo de servicio $\leq$ a 20 años. 66,67\% pertenecieron a los departamentos de Medicina y Emergencia. De los TS afectados, 72,92\% fueron de las áreas clínicas, $11,45 \%$ de áreas quirúrgicas y 3,13\% pertenecieron a servicios auxiliares y de apoyo diagnóstico. ConclusIones. La tasa habitual de TP en TS del HNDM fue aproximadamente 4,22 veces mayor que la tasa de incidencia nacional. Los TS más afectados fueron el personal técnico auxiliar, los nombrados, los menores de 40 años, aquellos con tiempo de servicio menor de 20 años y los que laboraban en áreas clínicas, y más del $60 \%$ pertenecieron a los departamentos de Medicina y Emergencia.
\end{abstract}

Palabras Clave. Tuberculosis pulmonar, trabajador de salud, Mycobacterium tuberculosis. (DeCS)

\begin{abstract}
OвJECTIVE. Determine the epidemiological profile of health care workers (HCW) affected by pulmonary tuberculosis (PT) in Dos de Mayo National Hospital (DMNH), Lima, Peru. Material and Methods. An observational, descriptive and retrospective study. The population were HCW diagnosed with PT in Dos de Mayo National Hospital. Epidemiological variables were collected: Age, gender, comorbidity, occupational group, service or department they belong, working situation, time of service, among others. For quantitative variables the mean, standard deviation, range and lower and upper value were determined, for qualitative variables the frequency analysis
\end{abstract}

I. Médico internista. Facultad de Medicina, Universidad Nacional Mayor de San Marcos Hospital Nacional Dos de Mayo, Lima.

2. Médico de personal. Hospital Nacional Dos de Mayo, Lima.

3. Médico intensivista. Facultad de Medicina, Universidad San Martín de Porres. Hospital Nacional Dos de Mayo, Lima. like absolute and relative frequencies were used. Incidence rates for 100000 was determined and statistical tests of central tendency and dispersion were used; $p$ value $<0,05$ was considered as significant. Results. The usual incidence rate of PT was 348 per 100000 . The most affected group was the auxiliary technical staff $(23,96 \%)$. The average age was 35,52 year-old $( \pm 10,22)$ and $70,83 \%$ were under of 40 year-old. The average time of hospital work was 9,23 years $( \pm$ 7,32); $91,70 \%$ had service time $\leq 20$ years; $66,67 \%$ belonged to the departments of Medicine and Emergency; $72,92 \%$ of the affected HCW, were from the clinical areas; $11,45 \%$ of surgical areas and 3,13\% belonged to auxiliary and diagnostic support services. ConcLusions. The usual rate of PT of HCW in the HNDM was approximately 4,22 times higher than the national incidence rate. The most affected $\mathrm{HCW}$ were auxiliary technical staff, appointed workers, age under of 40 years old, with service time under 20 years, health care workers that work in clinical areas and more than $60 \%$ belonged to the medicine and emergency departments.

KEYWORDS. Pulmonary tuberculosis, health care worker. (MeSH) 


\section{INTRODUCCIÓN}

Cada año en Perú se notifican aproximadamente 31 mil casos nuevos de tuberculosis (TB). Para 2016, la Organización Mundial de la Salud (OMS), a través de su reporte anual, estimó una incidencia de TB de 117 x 100000 habitantes (90-148). Ese mismo año se notificaron 31079 casos de TB a nivel nacional y el presupuesto nacional asignado para la TB fue de 101 millones de dólares americanos.

La incidencia anual de TB en trabajadores de salud (TS) de diferentes países en vías de desarrollo varía de 25 a 5361 por 100000 . La magnitud del riesgo de infección varía según el tipo de establecimiento, grupo ocupacional, prevalencia de la TB en la población, proporción de pacientes hospitalarios que reciben atención y la eficacia de las medidas de control de TB en el hospital. La probabilidad de infectarse de los TS depende del grado y el tiempo de contacto con el bacilo. Se describen ambientes hospitalarios, donde existe mayor probabilidad de infectarse con el bacilo: salas de emergencia, medicina y neumología. Así mismo, en los ambientes donde se realizan broncofibroscopia, cultivos de micobacterias, nebulización y necropsias. El riesgo de adquirir la enfermedad tiene una asociación evidente con la ocupación o profesión del TS, en especial, los profesionales asistenciales, quienes permanecen mayor tiempo en contacto con los enfermos. ${ }^{1}$

La incidencia de TB en la población general ha descendido progresivamente desde la década de 1990 hasta el año 2003, a un ritmo de $8 \%$ anual, mientras que desde 2003 hasta 2011 se ha mantenido estacionaria o presenta tendencia a aumentar, con una tasa entre 100 y 120 casos x 100000 habitantes. Lima es el departamento del país con mayor incidencia de TB, con tasas de incidencia de 479,7/100 000 habitantes. ${ }^{1}$ Alrededor del $50 \%$ a $60 \%$ de todos los casos de TB del país ocurren en Lima, obviamente, la gran población de la capital (9 millones 320 mil habitantes, según cifras del INEI, 2017), la elevada carga migratoria, el hacinamiento generalizado (viviendas, hospitales, centros penitenciarios, transporte), el incremento de casos de infección por VIH (a inicios de la década de 1980), convierten a la ciudad de Lima en una ciudad vulnerable, no solo para la infección y propagación de TB, sino también para la diseminación de otras enfermedades de transmisión aérea.

Según el reporte del Ministerio de Salud (Minsa) de 2011 y del análisis ejecutivo nacional de EsSalud de
2015, de todos los casos de TB en TS, el $57 \%$ procedían de centros hospitalarios del Minsa; $36 \%$, de Essalud; $7 \%$, de otras instituciones como hospitales de fuerzas armadas, clínicas privadas, etc. Así mismo, se informó que el personal de enfermería era el grupo de mayor riesgo, con una prevalencia de $27 \%$ en técnicos en enfermería; $18 \%$ en licenciadas en enfermería; y $17 \%$ en médicos. En la actualidad, se han incrementado los casos de TB en TS, según datos reportados por el Minsa, para el período 2013-2014. Del total de casos notificados, el $68 \%$ de casos trabajaban en establecimientos de salud del Minsa; $21 \%$, en EsSalud; $11 \%$, en hospitales de las fuerzas armadas y la policía nacional. Se encontró que el $83 \%$ de los TS afectados por TB fueron profesionales o técnicos de la salud asistencial, y, de estos, la mayor proporción de afectados fueron técnicos en enfermería, enfermeras y médicos. ${ }^{4}$

Cada año, alrededor de 200 TS adquieren la enfermedad de TB a nivel nacional. En el estudio realizado por Alarcón y col., se encontró alrededor de mil casos de TB a nivel nacional con una tendencia creciente en el estudio de seis años realizado, de 2007 a 2012. ${ }^{5}$ Las tasas elevadas de infección y enfermedad tuberculosa tienen una distribución heterogénea entre los TS, afecta en mayor proporción a los trabajadores asistenciales que a los administrativos. Otro grupo de riesgo descrito, son los estudiantes, residentes y alumnos en formación de las diferentes carreras de salud. El Hospital Nacional Dos de Mayo (HNDM) alberga el 3\% de los casos nacionales de TB, cada año, son atendidos alrededor de 800 a 1200 pacientes con TB en todas sus formas, el $70 \%$ corresponden a TB pulmonar (TBP) y alrededor de 250 a 350 casos de TB son hospitalizados anualmente, constituyéndose en fuente importante de contagio para los TS.

Diversas investigaciones han determinado que el riesgo de infección por TB en los hospitales es de 2 a 50 veces más que en la población normal..$^{6-8}$ Las tasas elevadas de infección y enfermedad tuberculosa en hospitales peruanos representan una constante amenaza para los TS. Esta situación problemática ha motivado la realización de la presente investigación.

\section{MATERIAL Y MÉTODOS}

Diseño y población de estudio. Se realizó una investigación observacional, descriptiva y retrospectiva. La población fueron los TS diagnosticados y tratados de TBP de 2004 a 2015. 
Fueron excluidos los casos con TB extrapulmonar, casos no registrados en la base de datos del médico del personal, TS, menores de 18 años y con permanencia menor a un mes en el servicio donde laboraba.

Variables y mediciones. Se recogieron variables epidemiológicas: edad, sexo, comorbilidad, grupo ocupacional, estatus laboral (nombrado-contratado), tiempo de servicio, ambiente laboral, entre otras variables.

Análisis estadístico. La información obtenida de la base de datos fue almacenada y procesada con el paquete estadístico de SSPS versión 23.0. Se utilizaron técnicas paramétricas y no paramétricas, análisis de tendencia central y de dispersión. Se consideró el valor de $\mathrm{p}<0,05$ como estadísticamente significativo.

Consideraciones éticas. En todo momento de la investigación se mantuvo la confidencialidad de la información, se trabajaron exclusivamente con datos epidemiológicos, determinantes específicos y de importancia para determinar el perfil epidemiológico de TBP en los TS del HNDM.

\section{RESULTADOS}

De siete a diez TS por año son afectados de TBP en el HNDM. En el periodo de estudio, se reportaron 96 casos de TBP en TS. En la Figura 1, se observa las tasas de incidencia anual (x 100000 TS). La tasa habitual de TB fue 348 por 100000 TS. Esta tasa de incidencia fue aproximadamente 4,22 veces mayor que la tasa nacional notificada el 2016, según reporte del Minsa $(86,4 \times 100000)$.

El 53,12\% (51/96) de los TS afectados de TB fueron bacilíferos (BK: positivo); 8,30\% (8/96) presentaron TB multidrogorresistente (MDR): 3 casos se reportaron en el servicio de Emergencia, 2 en Medicina, 2 en Pediatría
Tabla I. Comorbilidad en los trabajadores de salud del Hospital Nacional Dos de Mayo. 2004-2015

\begin{tabular}{lcr}
\hline Comorbilidad & Casos & $(\%)$ \\
\hline - Bronquitis crónica & 8 & 33,33 \\
- Diabetes mellitus & 6 & 25,00 \\
- Hipertensión arterial & 5 & 20,83 \\
- Conectivopatía & 4 & 16,67 \\
- Infección por VIH & 1 & 4,17 \\
Total & 24 & 100,00 \\
\hline
\end{tabular}

y 1 en el servicio de Cirugía. No hubo diferencia estadísticamente significativa para adquirir TB MDR según el servicio donde se labora $\left(\chi^{2}: 8,271\right.$, p: 0,30). El tiempo promedio para el diagnóstico de TB, desde el inicio de sus síntomas hasta el aislamiento del bacilo, fue 21,22 días ( $\mathrm{DE} \pm 6,86$ ), su valor mínimo fue 14 y el máximo, 31 días. El 52,10\% (50/96) de TS estaban nombrados. La edad promedio de los TS afectados de TBP fue 35,52 años (DE $\pm 10,22)$ y la población de 40 años o menos, 70,83\% (68/96). Los TS de sexo femenino fueron $58,63 \%(56 / 96)$, con discreta predominancia del sexo femenino, sin llegar a ser estadísticamente significativa (p: 0,07). El tiempo promedio de servicio (actividad laboral en años) en el grupo de estudio fue de 9,23 años (DE $\pm 7,32)$.

Uno de cada cuatro TS afectado de TBP presentaba algunas comorbilidades prevalentes (Tabla 1), como diabetes mellitus, bronquitis crónica y patología asmatiforme, hipertensión, conectivopatía e infección por VIH. Dentro del grupo de diabéticos, 83,34\% tuvieron DM2 y un caso DM1. En el grupo de las conectivopatías, todas fueron de sexo femenino, 2 TS padecían de LES, 1 de fibromialgia y 1 de artritis reumatoide.

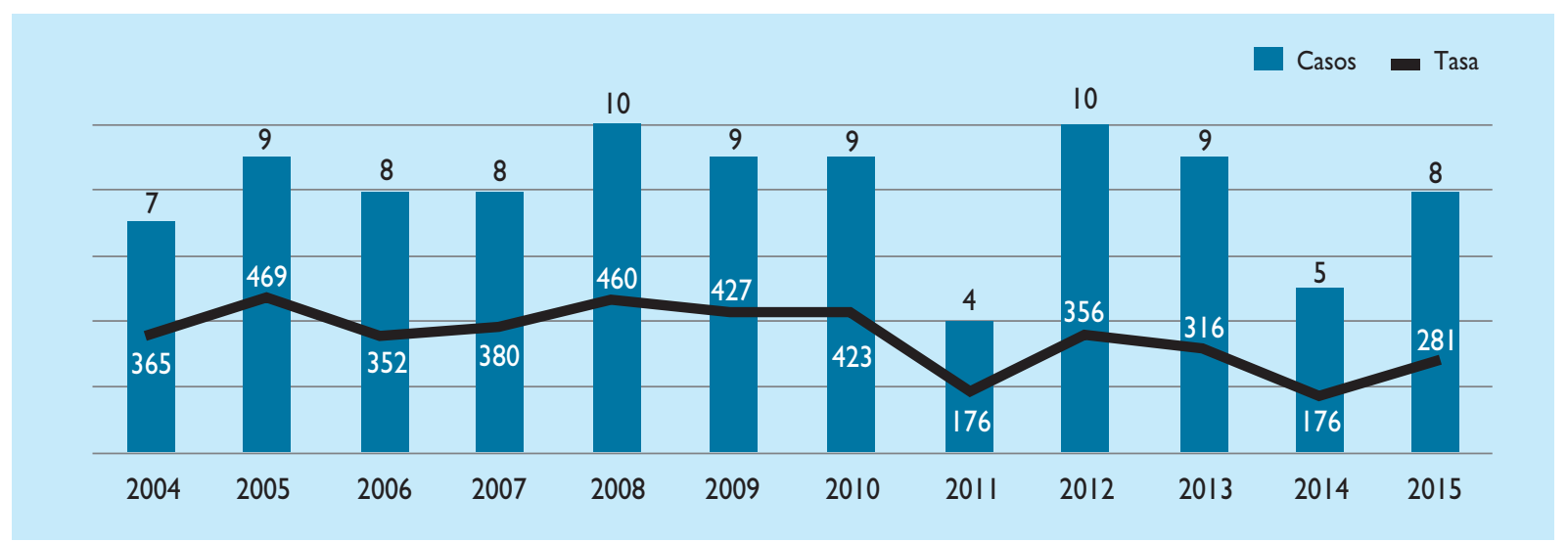

Figura I.Tasa de incidencia de tuberculosis pulmonar en trabajadores de salud. HNDM. 2004-20I5. 
El grupo ocupacional más afectado de la serie fue el personal técnico auxiliar $(23,96 \%)$ con 23 casos, posiblemente por el contacto cercano y prolongado con pacientes bacilíferos, en las salas de hospitalización y emergencia. Seguido de los médicos asistentes $(19,79 \%)$, residentes $(16,67 \%)$, internos $(15,63 \%)$, enfermeras $(12,50 \%)$. En el grupo de otros (11 casos), fueron incluidos otros TS, como obstetra, químico farmacéutico, psicóloga, tecnólogo y laboratorista $(5,20 \%)$, además de personal de servicio $(3,13 \%)$ y personal administrativo $(3,13 \%)$.

El 66,67\% de los TS afectados por TBP pertenecieron a los departamentos de Medicina y Emergencia, en estos dos departamentos se presentaron 64 casos (Tabla 2). El riesgo de contraer TBP en los TS que laboran en los departamentos mencionados fue de 1,6 veces más que los trabajadores de otros departamentos hospitalarios. Probablemente, este hallazgo está relacionado con que en estos departamentos se hospitalizan pacientes sintomáticos respiratorios y muchas veces $\sin$ diagnóstico conocido. Esto convierte prácticamente a estos departamentos en ambientes hospitalarios de alto riesgo, que constituyen una fuente importante de contagio por Micobacterium tuberculosis.

El 72,92\% de los casos de TBP de los TS se produjeron en áreas clínicas (medicina, emergencia y pediatría), donde las dos primeras tuvieron carga alta de TB. Las áreas quirúrgicas tuvieron riesgo intermedio, en estos servicios se produjo el $11,45 \%$ de los casos. Los servicios auxiliares de mantenimiento, limpieza y servicios administrativos resultaron servicios de bajo riesgo, con $3,13 \%$ de casos.

Tabla I. Tuberculosis pulmonar en trabajadores de salud en los departamentos y servicios del Hospital Nacional Dos de Mayo. 2004-2015

\begin{tabular}{lcr}
\hline Departamento/servicio & Casos & $(\%)$ \\
\hline - Medicina & 44 & 45,83 \\
- Emergencia & 20 & 20,83 \\
- Cirugía & $1 \mathrm{I}$ & $1 \mathrm{II}, 46$ \\
- Pediatría & 6 & 6,25 \\
- Ginecoobstetricia & 5 & 5,20 \\
- Servicio de Apoyo diagnóstico & 4 & 4,17 \\
- Servicio de Mantenimiento y vigilancia & 3 & 3,13 \\
- Servicio de Administración & 3 & 3,13 \\
Total & 96 & 100,00 \\
\hline
\end{tabular}

\section{DISCUSIÓN}

La TB ocupa el décimo lugar como causa de hospitalización en el HNDM, representa el 1,89 \% del total de las hospitalizaciones y es responsable del $11 \%$ de la mortalidad global en pacientes hospitalizados. El 84,54\% del total de trabajadores fue personal del equipo asistencial (médicos, enfermeras, técnicos de enfermería, residentes e internos) y el 15,46\% restante correspondieron al personal administrativo, personal de servicio y personal de apoyo diagnóstico. Se observó una tendencia creciente del número de TS a través de los años 2004 (1 855 TS) a 2015 (2 844), fenómeno esperado, probablemente, por la necesidad de incrementar los TS frente al incremento de la demanda.

El sexo femenino predominó discretamente (56/96). Estos hallazgos $(58,3 \%)$ fueron similares a los encontrados por Nakandakari y col. (58,9\% para el sexo femenino), en la investigación realizada en el Hospital Nacional Hipólito Unanue de Lima, ${ }^{9}$ pero contrastan con los hallazgos de Llerena y col., que encontraron cifras de 64,8\% (83/128), para el sexo femenino en el estudio realizado en Bogotá, Colombia. ${ }^{10}$ Esta discreta predominancia relativa del sexo femenino no fue estadísticamente significativa. (p :0,77). Estos hallazgos encontrados en el HNDM, probablemente, están relacionados con el mayor número de técnicas de enfermeras trabajando en el hospital, quienes son predominantemente del sexo femenino. La relación de técnicos de enfermería y médicos (población masculina predominante) fue de 1,58 al iniciar el estudio y de 1,54 en 2015. Por otro lado, la relación femenino/masculina del personal nombrado fue a favor del sexo femenino (F/M: 1,80). Los resultados sugieren que la TBP en TS del HNDM probablemente sea de predominancia femenina parcial o que no exista diferencias entre los sexos. Se requieren más estudios al respecto para emitir conclusiones contundentes.

Los TS que resultaron ser bacilíferos fue $53,12 \%$ (51/96), hallazgo importante para el diagnóstico precoz y oportuno. Sin embargo, $46,88 \%$ de los casos los estudios de baciloscopia fueron negativos, lo que retardó el diagnóstico y constituyeron un foco de diseminación intrahospitalario. Resultados similares encontraron Fica y col., los que reportaron una población bacilífera de $57,2 \%$ en su investigación realizada en TS de Santiago, Chile. ${ }^{11}$ Contrariamente, Anoop y col. encontraron baciloscopia positiva en 76,2\% de los TS con TBP en un estudio realizado en el sur de la India. Este último 
hallazgo, probablemente está relacionado a la mayor incidencia y gravedad de la TB en la India. ${ }^{12}$

El grupo ocupacional más afectado de TBP en TS del HNDM fueron técnicos de enfermería $(23,96 \%)$, seguido de los médicos asistentes $(19,76 \%)$, residentes $(16,67 \%)$ e internos $(15,63 \%)$. Estos resultados concuerdan con el reporte de Fica y col., quienes encontraron predominancia del grupo ocupacional de técnicos de enfermería (35,7\%), seguido de licenciadas de enfermería (14,3\%). El grupo ocupacional técnicos de enfermería ocupa el primer o segundo lugar como grupo ocupacional más afectado en diferentes reportes publicados. ${ }^{9,10,13}$ Recientemente, se ha reportado, según estadística nacional, que el grupo de técnicos de salud constituye el $66,6 \%$ de todos los caso reportados de TB en los TS. Esta elevada incidencia en este grupo ocupacional probablemente está relacionada con la modalidad de trabajo que ellos desarrollan. Ellos administran el tratamiento directamente observado y supervisado (DOTS). En los ambientes hospitalarios, los técnicos de enfermería trabajan en turnos mayores a 8 horas de trabajo con altas probabilidades de contagio. Finalmente, el riesgo incrementado de contraer TBP en los técnicos de enfermería probablemente está relacionado con su situación económica, pues, la remuneración económica en este grupo ocupacional es el más bajo de todo el equipo asistencial de salud.

Se encontró mayor frecuencia de TBP en TS nombrados $52,08 \%$ vs. $47,02 \%$ en contratados. A pesar de la aparente predominancia de TB en trabajadores nombrados en el grupo de casos no se encontró diferencias estadísticamente significativas ( $\mathrm{p}: 0,10)$. Estos resultados, difieren con los encontrados por Nakandakari y col., que reportaron predominancia de TB activa en el personal contratado $48 \%$ vs. $14 \%$ nombrado. ${ }^{9}$ Esta predominancia, en el personal contratado, también fue encontrada por Soto Cabezas y col., 47,4\% para el grupo de contratados vs. $28,3 \%$ nombrados. ${ }^{13}$ Estas diferencias probablemente se deban a que la mayoría de los TS del HNDM estaban nombrados en el periodo del estudio. Se encontró una relación entre nombrados/contratados de 3,20.

La tasa incrementada de incidencia de TB en técnicos auxiliares fue reportada desde hace muchos años en diferentes investigaciones a nivel mundial y específicamente en América Latina. ${ }^{14-16}$ La tasa de enfermedad TB activa en nuestro estudio osciló entre 197 y 789 x 100000 TS. Se alcanzó la menor tasa en 2008 y la más alta en 2010. Díaz y col., en una investigación realizada en el Hospital Docente de Cuba, encontraron que el riesgo de TB en personal técnico de enfermería era 8,21 veces mayor al grupo de enfermeras. ${ }^{16}$ En el presente estudio, solo doce enfermeras $(12,50 \%)$ adquirieron la enfermedad. Este hallazgo, probablemente, estaría relacionado al menor tiempo de contacto de las enfermeras con el paciente dentro de su labor rutinaria, comparado con los técnicos de salud, residentes e internos. La mayor tasa de incidencia de TB clínica registrada en las enfermeras en nuestra investigación fue 741 x 100000 en 2007. Sin embargo, existen reportes contrarios que describen mucho más mayor riesgo de TB en personal de enfermería, incluso mayor que los técnicos de enfermería y los médicos. ${ }^{17,18}$

La edad promedio, relativamente $(35,52 \mathrm{DE} \pm 10,22)$, joven encontrada es similar a la gran mayoría de estudios, incluso, se reporta como factor de riesgo para TB en TS. ${ }^{19,20}$ En el presente estudio, se encontró que el 70,83\% (68/96), de la población con TBP tuvieron 40 o menos años. Este hallazgo parece ser una constante en los diferentes reportes publicados. Por lo contrario, un estudio realizado en China (uno de los 22 países con alta carga de TB en el mundo), por Wang y col., encontró resultados diferentes. Mediante análisis multivariado, reportaron la edad de 51 años o más como factor de riesgo para TB. ${ }^{21}$ Esta diferencia, probablemente, está relacionada al rango amplio de la edad de los participantes (18 a 81 años), población heterogénea (estudio multicéntrico incluyeron TS de hospitales comunitarios, prefecturales y provinciales), población predominante femenina $(64,90 \%)$, quienes tienen mayor expectativa de vida en China y mayor proporción de mayores de edad en la población estudiada $(68,20 \%$ de la población tenían edad de 30 a más años). Adicionalmente, la disminución natural del sistema inmune a mayor edad jugaría un rol importante, pues la aparición de la enfermedad activa depende exclusivamente de la integridad del estado inmunológico del huésped.

Algunos factores asociados a enfermedad tuberculosa son el número de pacientes atendidos al año, estado clínico del paciente con TB, periodo o fase de tratamiento, ocupación de los TS, el lugar o servicio de trabajo, deficiencia en los sistemas de ventilación, ausencia de salas de aislamiento, deficientes uso de las barreras de barreras de protección personal y 
finalmente la presencia de alguna comorbilidad..$^{22} \mathrm{La}$ presencia de comorbilidad estuvo presente en el $25 \%$ de los TS con TB. La mayoría de ellos padecía de bronquitis crónica y asma bronquial además de otras enfermedades prevalentes descritas previamente. La presencia de estas enfermedades determina suficiente grado de inmunosupresión, como para incrementar el riesgo de enfermedad tuberculosa en este grupo, por lo que estos trabajadores deben ser reubicados en áreas de bajo riesgo para TB.

El 91,70\% de los TS estaban trabajando por un tiempo menor de 20 años. Este hallazgo comprueba que la TBP afecta predominantemente a personas relativamente jóvenes y con tiempo de servicio relativamente menor.

En conclusión, la tasa habitual de TBP en los TS del HNDM fue aproximadamente 4,22 veces mayor que la tasa de incidencia nacional. Los trabajadores más afectados fueron el personal técnico auxiliar, los nombrados, los menores de 40 años, aquellos con tiempo de servicio menor de 20 años, los que laboran en áreas clínicas; más del $60 \%$ pertenecieron a los departamentos de Medicina y Emergencia.

\section{REFERENCIAS BIBLIOGRÁFICAS}

I. World Health Organization WHO, Global Tuberculosis Report. Ginebra: WHO; 2017.

2. Chugh TD. Burden of nosocomial tuberculosis in healthcare workers in India. Current medicine research and practice. 2017;7(I):18-19.

3. Ministerio de Salud del Perú. Análisis de la Situación de Salud del Perú. MINSA. Dirección General de Epidemiologia, Lima, Perú. 2013.

4. Ministerio de salud del Perú.Análisis de la situación epidemiológica de la tuberculosis en el Perú. 2015.

5. Alarcón V, Alarcón E, Figueroa C, Mendoza-Ticona A. Tuberculosis en el Perú: situación epidemiológica, avances y desafíos para su control. Rev Per Med Experim Salud Pública. 2017;34:299-310.

6. Accinelli R, Álvarez L,Valles, Guio H, Muñoz P.Annual risk of Tuberculosis infection among medical student of Universidad Peruana Cayetano Heredia. Lima Perú: American Thoracic Society Meeting, 2002. Atlanta MA,ATs [Abstract Poster \#912].

7. Jiamjarasrangsi W, Hirunsuthikul N, Kamolratana-kul P. Tuberculosis among health care workers at King Chulalongkorn Memorial Hospital, 1988-2002. Int J Tuberc Lung Dis. 2005;9: I253-8.

8. Baussano I, Nunn P,Williams B, Pivetta E, Bugiani M, Scano F.Tuberculosis among health care workers. Emerg Infect Dis. 201 I; I7(3):488-94.
9. Nakandakari M, De la Rosa D, Gutiérrez J, Bryson W. Tuberculosis en trabajadores de salud: Estudio epidemiológico y clínico en el Hospital Nacional Hipólito Unanue. Rev Med Hered. 20I4; 25: 129-34.

10. Llerena C, Zabaleta A. Evaluación por el laboratorio de los casos de tuberculosis en profesionales del área de la salud. Acta Medica Colombiana. 2014;39(4):321-326.

II. Fica A, Ramonda P, Jemenao M, Zambrano A, Cifuentes M, Febré N, et al. Tuberculosis in health care workers from a public health service in Santiago, Chile. Rev Chil Infectol. 2009;26(I):34-8.

12. Anoop M, Thambu D, Kurien T, Kurivilla P, Balaji V, Jesudason M, Prassana M. Risk factors for tuberculosis among health care workers in South India:A nested case-control study. Journal of Clinical Epidemiology. 2013; 66:67-74

13. Soto-Cabezas M, Chávez-Pachas A, Arrasco-Alegre J, Yagui-Moscoso M. Tuberculosis en trabajadores de salud en el Perú 2013-2015. Rev Perú Med Exp Salud Publica. 2016;33:607-6I5.

14. McKenna M, Hutton M, Cauthen G, Onorato M,The Association between occupation and tuberculosis.A population-based survey.Am J Respir Crit Care Med. 1996; 154: 587-93.

I5. Naidoo S, Jinabhai C. Tuberculosis in health worker in KwaZulu-Natal, South Africa. Int J Lung Dis. 2006; 10:676-82.

16. Diaz Castrillo A, Dueñas Mojena D, Lazo-Álvarez M.A, BorrotoGutiérrez S, Gonzales-Ochoa E. Tuberculosis en trabajadores de salud un hospital psiquiátrico de la Habana. 1997-2003. Rev Panam Infectol. 2005;7:22-6.

17. González C, Araujo G, Agoglia R, Hernández S, Seguel I, Sáenz C. Tuberculosis en trabajadores de salud. Medicina. 20I5 [Internet]. Feb 2010 [citado 28 Ago 20I5];70(I):23-30. Disponible en: http:// medicinabuenosaires.com/demo/revistas/vol70-10/I/v70_nI_p23_30. pdf

18. Borroto Gutiérrez S, Sevy Court J, Fumero Leru M, González Ochoa E, Machado Molina D. Riesgo de ocurrencia de la tuberculosis en los trabajadores del Hospital Universitario Neumológico Benéfico de la Habana. Rev Cub Med Trop. 2012;64(I):55-60.

19. Tudor C, Van Der Walt M, Bruce M, Dorman S, Pan W, Yenokyan G, Farley J. Occupational risk factors for tuberculosis among healthcare workers in KwaZulu-Natal. South Africa. Clin Infect Dis. 2016;62(Suppl 3): S255-S26I.

20. Riboty Lara A. Factores de riesgo ocupacionales y no ocupacionales para enfermar de tuberculosis pulmonar en trabajadores de salud-HNGAI. 1995-2000. tesis de Posgrado, 2005. Universidad Nacional Mayor de San Marcos, Lima, Perú.

21. Xiao-Ning Wang, Tian-Lun He, Meng-Jie Geng, Yu-Dan Song, Ji-Chun Wang, et al. Prevalence of and risk factors for tuberculosis among healthcare workers in Chinese tuberculosis facilities. Infectious Diseases of Poverty. 2018;7(26);3-I I. https://doi.org/I0.I I86/s40249-0I8-0407-6

22. Barboza A, Peña O,Valderrama A, Restrepo $H$. Factores de riesgo para tuberculosis en trabajadores de servicios de urgencias, en dos niveles de atención de salud. Rev Col Salud Ocupac. 20I4; 4(2):30-33.

CoRRESPONDENCIA: Carlos Contreras ccontrerashndm@hotmail.com

DeCLARACIÓN DE CONFLICTO DE INTERÉs: Ninguno, según los autores. FINANCIAMIENTO: Por los autores.

FECHA DE RECEPCIÓN: 21 de marzo de 2019.

FECHA DE ACEPTACIÓN: 30 de marzo de 2019. 